See discussions, stats, and author profiles for this publication at: https://www.researchgate.net/publication/314279522

\title{
A novel four-dimensional angiographic approach to assess dynamic superficial wall stress of coronary arteries in vivo: Initial experience in evaluating vessel sites with subsequent...
}

Article in Eurolntervention: journal of EuroPCR in collaboration with the Working Group on Interventional Cardiology of the European Society of Cardiology · March 2017 DOI: 10.4244/EIJ-D-16-01020

CITATIONS

7 authors, including:

Xinlei Wu

Shanghai Jiao Tong University

16 PUBLICATIONS 100 CITATIONS

SEE PROFILE

Yingguang Li

Leiden University

24 PUBLICATIONS 240 CITATIONS

SEE PROFILE
READS

71

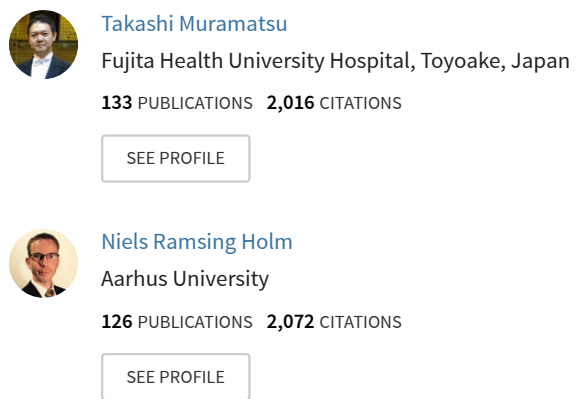

Some of the authors of this publication are also working on these related projects:

In vivo magnetic resonance imaging and spectroscopy of Alzheimer's disease in transgenic mice View project

PLATO Study View project 


\title{
A novel four-dimensional angiographic approach to assess dynamic superficial wall stress of coronary arteries in vivo: initial experience in evaluating vessel sites with subsequent plaque rupture
}

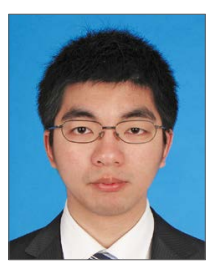

Xinlei $\mathbf{W u}^{1}, \mathrm{MSc}$; Clemens von Birgelen ${ }^{2,3}, \mathrm{MD}, \mathrm{PhD}$; Takashi Muramatsu', MD, PhD; Yingguang Li ${ }^{5}$, MSc; Niels Ramsing Holm6, MD; Johan H.C. Reiber ${ }^{5}$, PhD; Shengxian $\mathrm{Tu}^{1,5 *}, \mathrm{PhD}$

1. Med-X Research Institute, School of Biomedical Engineering, Shanghai Jiao Tong University, Shanghai, China; 2. Department of Cardiology, Thoraxcentrum Twente, Medisch Spectrum Twente, Enschede, the Netherlands; 3. Health Technology and Services Research, MIRA Institute, University of Twente, Enschede, the Netherlands; 4. Department of Cardiology, Fujita Health University Hospital, Toyoake, Japan; 5. Department of Radiology, Leiden University Medical Center, Leiden, the Netherlands; 6. Department of Cardiology, Aarhus University Hospital, Skejby, Denmark

X. Wu and C. von Birgelen contributed equally to this manuscript.

This paper also includes supplementary data published online at: http://www.pcronline.com/eurointervention/124th_issue/168

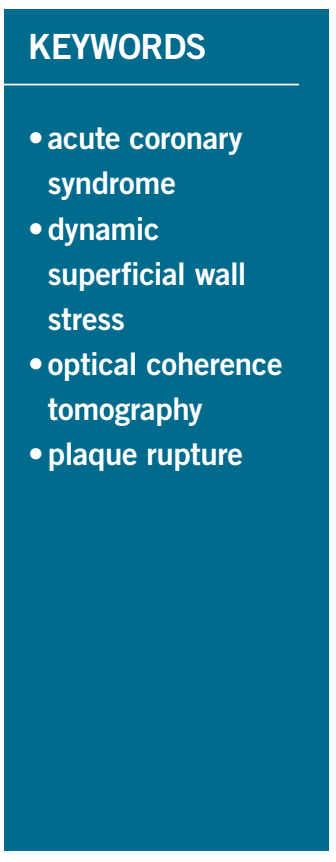

\begin{abstract}
Aims: Repetitive, fluctuating stress is an important biomechanical mechanism that underlies the rupture of atherosclerotic plaques. We developed a novel coronary angiography-based method for in vivo fourdimensional analysis of dynamic superficial wall stress (SWS) in coronary plaques and applied it for the first time in two clinical cases. Our aim was to investigate the potential relationship between dynamic stress concentration at baseline and plaque rupture during acute coronary syndrome (ACS) several months later.
\end{abstract}

Methods and results: Three-dimensional angiographic reconstructions of the interrogated arteries were performed at several phases of the cardiac cycle, followed by finite element analysis to obtain the dynamic SWS data. The peak stress at baseline was found at the distal and proximal lesion longitudinal shoulders, being $121.8 \mathrm{kPa}$ and $98.0 \mathrm{kPa}$, respectively. Intriguingly, in both cases, the sites with the highest SWS concentration at baseline co-registered with the location of plaque rupture during ACS, respectively six and 18 months after the baseline angiographic assessment.

Conclusions: A novel angiography-based analysis method for four-dimensional evaluation of dynamic SWS was feasible for investigating plaque biomechanical behaviour in vivo. Initial experience suggests that this technique could be useful in exploring mechanisms of future plaque rupture.

*Corresponding author: Med-X Research Institute, Shanghai Jiao Tong University, Room 123, No. 1954, Huashan Road, Shanghai200030,China.E-mail:sxtu@sjtu.edu.cn 


\section{Introduction}

Coronary plaque rupture is often caused by a tear in a fibrous cap that is weakened by inflammation and unable to resist mechanical stress which causes biomechanical fatigue ${ }^{1-3}$. Angiography does not depict plaques or provide information on vulnerability, but the dynamic image sequences provide valuable additional information on the cyclic movement of lumen contours. This characterises plaque deformation performance that might differ between vulnerable lesions and mechanically stable segments. We therefore developed a novel approach for four-dimensional (4D) biomechanical analysis under realistic displacement loads, combining finite element analysis with serial angiographic reconstructions, and assessed the relationship between stress concentrations in the superficial vessel wall and later location of plaque rupture.

\section{Methods}

This proof-of-concept study used coronary angiography recordings of two patients who underwent angiography and who, several months later, developed acute coronary syndromes (ACS), assessed with repeat angiography and optical coherence tomography (OCT). Patients were retrospectively identified from a database of Fujita Health University Hospital, Toyoake, Japan. The local ethics committee approved this study; patients provided written informed consent.

\section{CASE 1}

A 50-year-old man with a mild-to-moderate mid-LAD lesion (Figure 1, Moving image 1) showed deterioration to a severe stenosis after six months (Moving image 2). OCT revealed thrombi, plaque ruptures and a partially healed rupture at the distal lesion border.

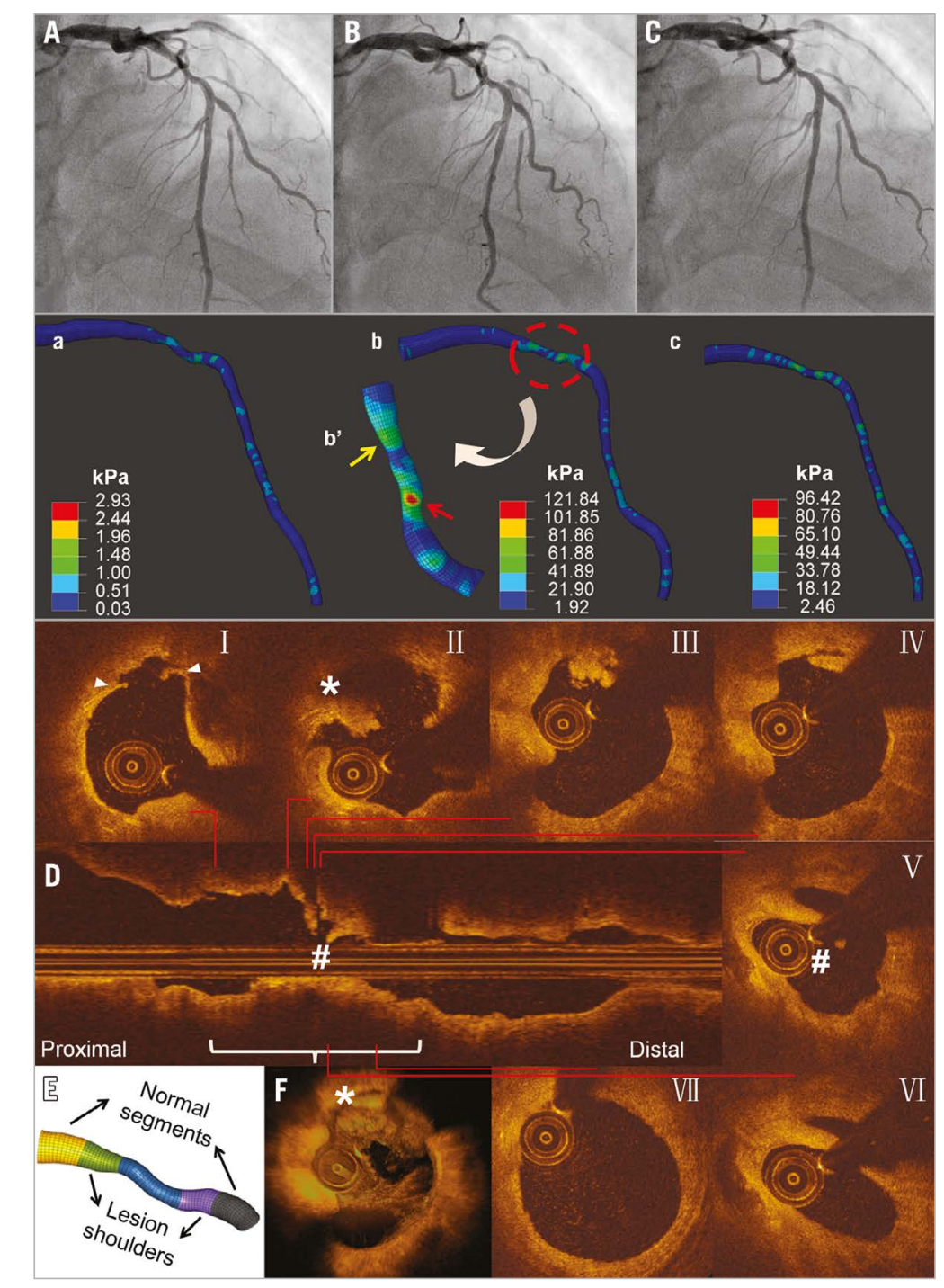

Figure 1. Angiography and SWS at baseline, and OCT imaging during ACS after six months (Case 1). Baseline angiography at different phases of the cardiac cycle (A-C) shows mild-to-moderate mid-LAD lesion (Moving image 1). SWS distributions at corresponding cardiac phases (a-c) reveal high values at mid-lesion and distal-lesion shoulder (Moving image 5) with more local SWS concentrations in the stenotic segment, particularly in distal lesion shoulder (arrow; flipped view b'). During ACS, transversal OCT images (I-VII) and longitudinal (D) and $3 D$ OCT reconstruction $(F)$ show thrombus and plaque rupture (I-IV), and partly healed ruptured plaque (V-VI). The spot with high SWS concentration at baseline (red arrow, b') corresponds with rupture site (II). E) Definition of segments of interest. 


\section{CASE 2}

A 69-year-old man with an intermediate mid-LCx lesion (Figure 2, Moving image 3) showed progression to a severe mid-LCx lesion 18 months later (Moving image 4). OCT showed thrombi.

Three-dimensional (3D) lumen geometries at different cardiac phases were reconstructed with QAngio XA 3D RE (Research Edition) (Medis Specials bv, Leiden, the Netherlands) ${ }^{4}$ and were used to perform finite element analysis (Supplementary Appendix 1). Historical data ${ }^{5}$ were utilised for material characteristics. Dynamic (4D) superficial wall stress (SWS) was calculated with Abaqus (V6.13, SIMULIA ${ }^{\text {TM}}$; Dassault Systèmes ${ }^{\circledR}$ Simulia Corp., Johnston, RI, USA). The von Mises stress, that takes into account all stresses in three principal axes, was used to report SWS.

\section{Results}

Angiographic reconstructions were performed at mid-diastole, end-diastole, and end-systole. In case 1, the reconstructed lesion segment showed the following cyclic changes from end-diastole to end-systole: segment length $104.3 \mathrm{~mm}-101.3 \mathrm{~mm}$; minimal lumen area $2.45 \mathrm{~mm}^{2}-2.15 \mathrm{~mm}^{2}$; and percent diameter stenosis $52 \%-55 \%$. In case 2, corresponding changes were: $112.1 \mathrm{~mm}-106.1 \mathrm{~mm}$; $3.32 \mathrm{~mm}^{2}-2.94 \mathrm{~mm}^{2}$; and $50 \%-52 \%$. The peak and time-averaged SWS were significantly higher in lesion segments than in nonculprit vessels (Supplementary Table 1). SWS measurements in non-culprit vessels were similar to those in reference segments.

In case 1 , SWS concentrations were highest at mid-lesion and distal lesion shoulder (Figure 1, Moving image 5). Peak SWS (121.8 $\mathrm{kPa}$ ) was found during end-systole (distal lesion shoulder). During

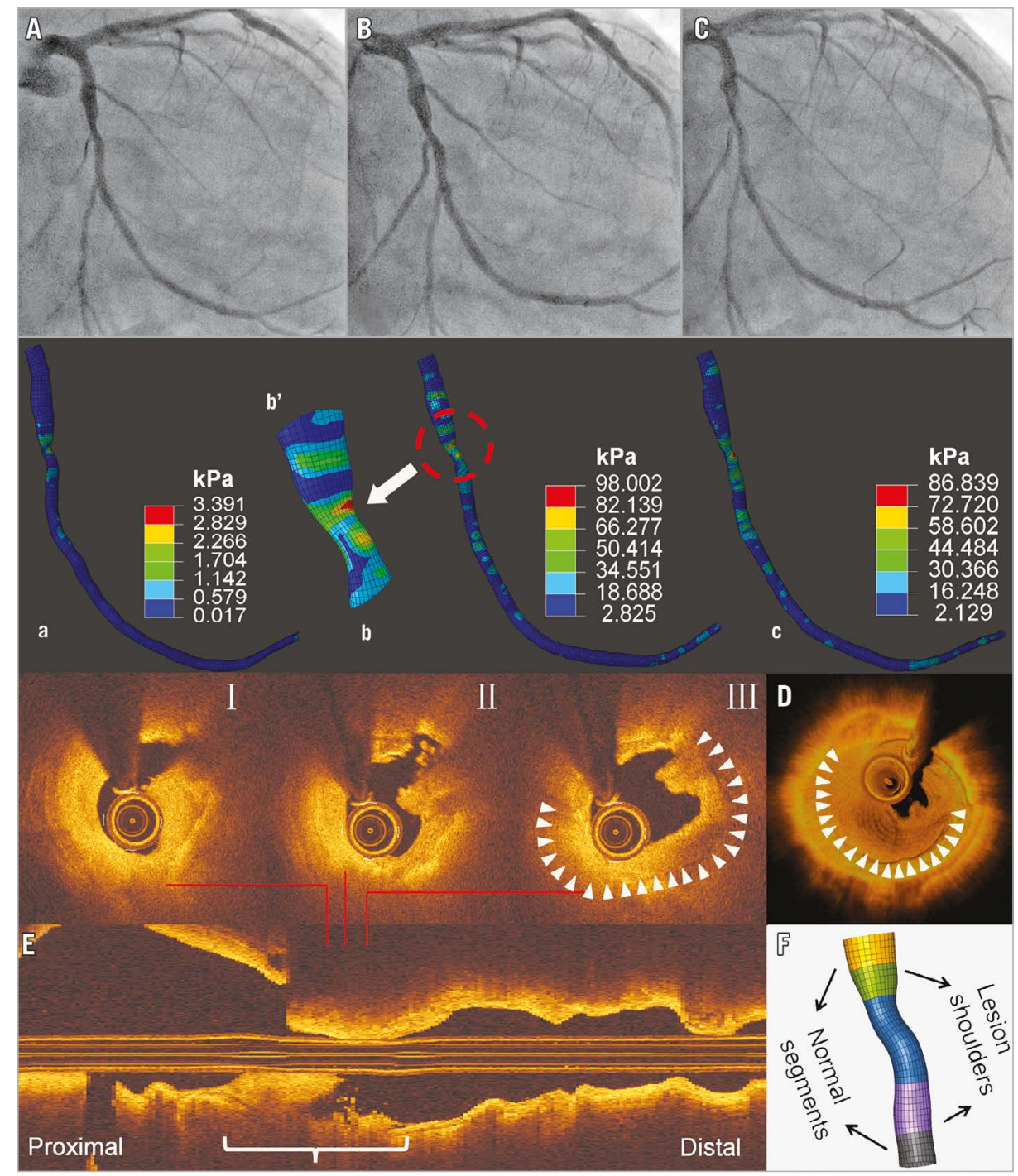

Figure 2. Angiography and SWS at baseline, and OCT imaging during ACS after 18 months (Case 2). Baseline angiography shows a mild-to-moderate mid-LCX lesion (A-C). SWS distributions at corresponding phases of the cardiac cycle (a-c) reveal high SWS at mid-lesion and proximal lesion shoulder (Moving image 6), with more local SWS concentrations in stenotic segment - mainly in proximal lesion shoulder (arrow; zoomed view b'). During ACS, OCT (I-III) and $3 D$ (D) and longitudinal OCT reconstructions (E) reveal lumen irregularity, thrombus, and plaque rupture. The spot with the highest SWS concentration at baseline corresponds with rupture site (I-III). F) Definition of segments of interest. 
the entire cardiac cycle, the distal lesion shoulder showed the highest time-averaged SWS (18.7 $\mathrm{kPa})$, followed by mid-lesion $(15.7 \mathrm{kPa})$. In case 2 (Figure 2, Moving image 6), more stress concentrations were observed at the proximal lesion shoulder: highest peak and time-averaged SWS were $98.0 \mathrm{kPa}$ and $23.5 \mathrm{kPa}$. In both cases, spots with the highest SWS at baseline co-registered with sites of subsequent rupture. Furthermore, locations of strain concentration in lesion segments were consistent with subsequent plaque rupture sites (Supplementary Figure 1). Further results are presented in Supplementary Appendix 2.

\section{Discussion}

This novel approach for the analysis of spatial and temporal variations in coronary anatomy by use of the finite element method can analyse dynamic SWS in vivo. In two clinical cases, sites with high SWS concentrations at baseline co-registered with sites of later plaque rupture.

Repetitive and fluctuating stress leads to material fatigue, and the balance between plaque structural stress and local strength of the superficial vessel wall determines where plaque rupture occurs. Some patient-specific computational methods have been proposed to compute plaque structural stress ${ }^{6-8}$. These approaches require plaque scanning and comprehensive data on the material properties of all plaque components ${ }^{9}$ and apply only static analyses of plaque structural stress, resulting in unrealistic representations of time-varying (cyclic) stress distribution.

Our present approach requires neither plaque segmentation nor data on complex plaque material properties or dynamic blood pressure changes. The rationale is as follows: the complex biomechanical and physiological interactions involving plaque material property, cyclic blood pressure, vessel stretching, bending, and twisting determine altogether the lumen change during cardiac contraction ${ }^{10}$. By subtracting changes in lumen dimensions over time and applying this in the finite element analysis (using the displacement loading method instead of the conventional mechanical force loading method ${ }^{5,11}$ ), our approach indirectly incorporates these complex biomechanical and physiological interactions in the analysis. This results in a practical approach to compute stress on the superficial arterial wall layer that, if present, includes the fibrous cap. We measured higher stress during systole at sites with abrupt changes in lumen geometry and (presumably) material properties. The results cannot be extended into the plaque core; nevertheless, they may be most relevant to assess stress distribution on fibrous caps ${ }^{12}$. Future research may address the natural history of coronary disease and the effects of novel drugs, bioresorbable scaffolds or stents on biomechanical vessel wall stability $^{13,14}$. See Supplementary Appendix 3 for limitations.

\section{Conclusions}

A novel angiography-based analysis method for 4D evaluation of dynamic SWS was feasible for investigating the plaque biomechanical behaviour in vivo. Initial experience suggests that it could be useful for exploring mechanisms of future plaque rupture.

\section{Impact on daily practice}

This novel approach for 4D biomechanical analysis of spatial and temporal variations in the coronary anatomy allows the assessment of dynamic superficial wall stress in vivo. It might be useful for investigating mechanisms of plaque rupture.

\section{Acknowledgements}

S. Tu acknowledges support by The Program for Professor of Special Appointment (Eastern Scholar) at Shanghai Institutions of Higher Learning (No. TP2015014) and the Shanghai Pujiang Program (No. 15PJ1404200).

\section{Funding}

This work was supported by the National Key Research and Development Program of China (No. 2016YFC0100500) and the National Natural Science Foundation of China (No. 31500797 and 81570456).

\section{Conflict of interest statement}

C. von Birgelen has received lecture fees from AstraZeneca; his institution has received research grants, provided by AstraZeneca, Biotronik, Boston Scientific, and Medtronic. Y. Li is an employee of Medis. N. Holm has received speaker fees from St. Jude Medical, Biotronik and Terumo, and institutional research grants from St. Jude Medical, Terumo, Boston Scientific, Medtronic, Biotronik, Medis and Cordis. J. Reiber is the CEO of Medis. S. Tu has received a research grant from Medis. The other authors have no conflicts of interest to declare.

\section{References}

1. von Birgelen C, Klinkhart W, Mintz GS, Papatheodorou A, Herrmann J, Baumgart D, Haude M, Wieneke H, Ge J, Erbel R. Plaque distribution and vascular remodeling of ruptured and nonruptured coronary plaques in the same vessel: an intravascular ultrasound study in vivo. J Am Coll Cardiol. 2001;37:1864-70.

2. Versluis A, Bank AJ, Douglas WH. Fatigue and plaque rupture in myocardial infarction. $J$ Biomech. 2006;39:339-47.

3. Brown AJ, Teng Z, Evans PC, Gillard JH, Samady H, Bennett MR. Role of biomechanical forces in the natural history of coronary atherosclerosis. Nat Rev Cardiol. 2016;13:210-20.

4. Tu S, Barbato E, Köszegi Z, Yang J, Sun Z, Holm NR, Tar B, Li Y, Rusinaru D, Wijns W, Reiber JH. Fractional flow reserve calculation from 3-dimensional quantitative coronary angiography and TIMI frame count: a fast computer model to quantify the functional significance of moderately obstructed coronary arteries. JACC Cardiovasc Interv. 2014;7:768-77.

5. Huang H, Virmani R, Younis H, Burke AP, Kamm RD, Lee RT. The impact of calcification on the biomechanical stability of atherosclerotic plaques. Circulation. 2001;103:1051-6.

6. Ohayon J, Finet G, Gharib AM, Herzka DA, Tracqui P, Heroux J, Rioufol G, Kotys MS, Elagha A, Pettigrew RI. Necrotic core thickness and positive arterial remodeling index: emergent 
biomechanical factors for evaluating the risk of plaque rupture. $\mathrm{Am}$ J Physiol Heart Circ Physiol. 2008;295:H717-27.

7. Tang D, Yang C, Zheng J, Woodard PK, Saffitz JE, Sicard GA, Pilgram TK, Yuan C. Quantifying effects of plaque structure and material properties on stress distributions in human atherosclerotic plaques using 3D FSI models. J Biomech Eng. 2005; 127:1185-94.

8. Teng Z, Canton G, Yuan C, Ferguson M, Yang C, Huang X, Zheng J, Woodard PK, Tang D. 3D critical plaque wall stress is a better predictor of carotid plaque rupture sites than flow shear stress: An in vivo MRI-based 3D FSI study. J Biomech Eng. 2010;132:031007.

9. Holzapfel GA, Mulvihill JJ, Cunnane EM, Walsh MT. Computational approaches for analyzing the mechanics of atherosclerotic plaques: a review. J Biomech. 2014;47:859-69.

10. Vanepps JS, Vorp DA. Mechano-pathobiology of atherogenesis: a review. J Surg Res. 2007;142:202-17.

11. Teng Z, Brown AJ, Calvert PA, Parker RA, Obaid DR, Huang Y, Hoole SP, West NE, Gillard JH, Bennett MR. Coronary plaque structural stress is associated with plaque composition and subtype and higher in acute coronary syndrome: the BEACON I (Biomechanical Evaluation of Atheromatous Coronary Arteries) study. Circ Cardiovasc Imaging. 2014;7:461-70.

12. Schieffer B, Schieffer E, Hilfiker-Kleiner D, Hilfiker A, Kovanen PT, Kaartinen M, Nussberger J, Harringer W, Drexler H. Expression of angiotensin II and interleukin 6 in human coronary atherosclerotic plaques. Potential implications for inflammation and plaque instability. Circulation. 2000;101:1372-8.

13. Haude M, Ince H, Abizaid A, Toelg R, Lemos PA, von Birgelen C, Christiansen EH, Wijns W, Neumann FJ, Kaiser C, Eeckhout E, Lim ST, Escaned J, Garcia-Garcia HM, Waksman R. Safety and performance of the second-generation drug-eluting absorbable metal scaffold in patients with de-novo coronary artery lesions (BIOSOLVE-II): 6 month results of a prospective, multicentre, non-randomised, first-in-man trial. Lancet. 2016;387:31-9.
14. von Birgelen C, Kok MM, van der Heijden LC, Danse PW, Schotborgh CE, Scholte M, Gin RM, Somi S, van Houwelingen KG, Stoel MG, de Man FH, Louwerenburg JH, Hartmann M, Zocca P, Linssen GC, van der Palen J, Doggen CJ, Löwik MM. Very thin strut biodegradable polymer everolimus-eluting and sirolimus-eluting stents versus durable polymer zotarolimus-eluting stents in allcomers with coronary artery disease (BIO-RESORT): a three-arm, randomised, non-inferiority trial. Lancet. 2016;388:2607-17.

\section{Supplementary data}

Supplementary Appendix 1. Methods.

Supplementary Appendix 2. Additional results.

Supplementary Appendix 3. Limitations.

Supplementary Table 1. Peak and time-averaged superficial wall stress (SWS) in culprit lesion and non-culprit vessel segments.

Supplementary Figure 1. Strain distribution of both cases at corresponding phases of peak stress at baseline.

Moving image 1. Coronary angiogram of the LAD lesion at baseline (Case 1).

Moving image 2. Coronary angiogram of the LAD lesion at the time of ACS episode (Case 1).

Moving image 3. Coronary angiogram of the LCx lesion at baseline (Case 2).

Moving image 4. Coronary angiogram of the $\mathrm{LCx}$ lesion at the time of ACS episode (Case 2).

Moving image 5. Dynamic superficial wall stress of the LAD lesion during cardiac contractions (Case 1).

Moving image 6. Dynamic superficial wall stress of the $\mathrm{LCx}$ lesion during cardiac contractions (Case 2).

The supplementary data are published online at:

http://www.pcronline.com/

eurointervention/124th_issue/168 


\section{Supplementary data}

\section{Supplementary Appendix 1. Methods}

3D lumen geometries of coronary arteries at the different cardiac phases were reconstructed from two angiographic projections with minimal vessel overlap and foreshortening, acquired several months before ACS, using QAngioXA 3D RE (Research Edition) (Medis Specials bv, Leiden, the Netherlands) [4].

All reconstructed lumen geometries were then uniformly discretised into the same structured meshes of quadrilateral elements with the same node seeds both at the longitudinal and circumferential directions. The total distance of the nodes on each circle at the circumferential direction from one configuration to another was calculated along the artery to determine one-to-one correspondence under the assumption of homogeneous continuum. To avoid the local stress concentration caused by poor mesh quality, these original meshes were then optimised by smoothing function with the criteria of shape parameters, including mesh Jacobian (0.85), aspect ratio (3.0), maximum angle $\left(>110^{\circ}\right)$ and minimum angle $\left(<70^{\circ}\right)$. According to the optimisation principle for minimum total displacement of all nodes moving from one configuration to the next, each node was implemented through one-to-one mapping between two adjacent configurations. The displacement load of each node was obtained by the change in its spatial locations during cardiac contraction. A thickness of $60 \mu \mathrm{m}$ for the superficial wall was applied, since accuracy in delineation of the lumen contours on angiographic images is half a pixel of $120 \mu \mathrm{m}$ in modern X-ray angiographic systems when exported into an image size of $1,024 \times 1,024$ pixels. The interval of cardiac phases (as used for the 3D angiographic reconstruction) was determined from the electrocardiogram that was recorded with angiographic runs. Finite element models were assumed as a homogeneous, isotropic, nearly incompressible, Mooney-Rivlin material that described different strain energy density functions by modulating two material parameters, i.e., $\mathrm{C}_{1}$ and $\mathrm{C}_{2}$. These two parameters were set as $2.6 \mathrm{kPa}$ and 8.4 for normal segments, and $5.1 \mathrm{kPa}$ and 13.0 for stenotic segments based on historical data [5].

Dynamic superficial wall stress (SWS) of cardiac contraction-induced variation in vessel wall morphology was calculated in Abaqus software (V6.13, SIMULIA; Dassault Systèmes ${ }^{\circledR}$ Simulia Corp., Johnston, RI, USA) using an input file which was the integration of finite element modelling and displacement load functions. To investigate the effect of dynamic SWS on plaque rupture, stress measurements in culprit segments were compared with measurements in non-culprit arteries of the same patient. The von Mises stress that takes into account all stresses in three principal axes was used to report SWS. 
Supplementary Table 1. Peak and time-averaged superficial wall stress (SWS) in culprit lesion and non-culprit vessel segments.

\begin{tabular}{|c|c|c|c|c|c|c|}
\hline & & & $\begin{array}{l}\text { Culprit } \\
\text { lesion } \\
\text { segment }\end{array}$ & & & $\begin{array}{c}\text { Non- } \\
\text { culprit } \\
\text { vessel } \\
\text { segment }\end{array}$ \\
\hline & $\begin{array}{c}\text { Proximal } \\
\text { normal } \\
\text { reference }\end{array}$ & $\begin{array}{l}\text { Proximal } \\
\text { lesion } \\
\text { longitudinal } \\
\text { shoulder }\end{array}$ & $\begin{array}{c}\text { Mid } \\
\text { lesion } \\
\text { portion }\end{array}$ & $\begin{array}{l}\text { Distal } \\
\text { lesion } \\
\text { longitudinal } \\
\text { shoulder }\end{array}$ & $\begin{array}{c}\text { Distal } \\
\text { normal } \\
\text { reference }\end{array}$ & $\begin{array}{l}\text { Normal } \\
\text { mid } \\
\text { coronary } \\
\text { segment }\end{array}$ \\
\hline \multicolumn{7}{|c|}{ Case 1} \\
\hline $\begin{array}{l}\text { Peak } \\
\text { sWS } \\
(\mathrm{kPa})\end{array}$ & 34.4 & 61.3 & 100.7 & 121.8 & 59.0 & 40.9 \\
\hline $\begin{array}{c}\text { Time- } \\
\text { averaged } \\
\text { sWS } \\
\text { (kPa) }\end{array}$ & 7.5 & 11.4 & 15.7 & 18.7 & 9.5 & 6.8 \\
\hline \multicolumn{7}{|c|}{ Case 2} \\
\hline $\begin{array}{l}\text { Peak } \\
\text { sWS } \\
(\mathrm{kPa})\end{array}$ & 51.2 & 98.0 & 97.5 & 49.7 & 21.1 & 36.0 \\
\hline $\begin{array}{c}\text { Time- } \\
\text { averaged } \\
\text { SWS } \\
\text { (kPa) }\end{array}$ & 11.5 & 23.5 & 16.9 & 15.0 & 6.4 & 4.3 \\
\hline
\end{tabular}




\section{Supplementary Figure 1.}

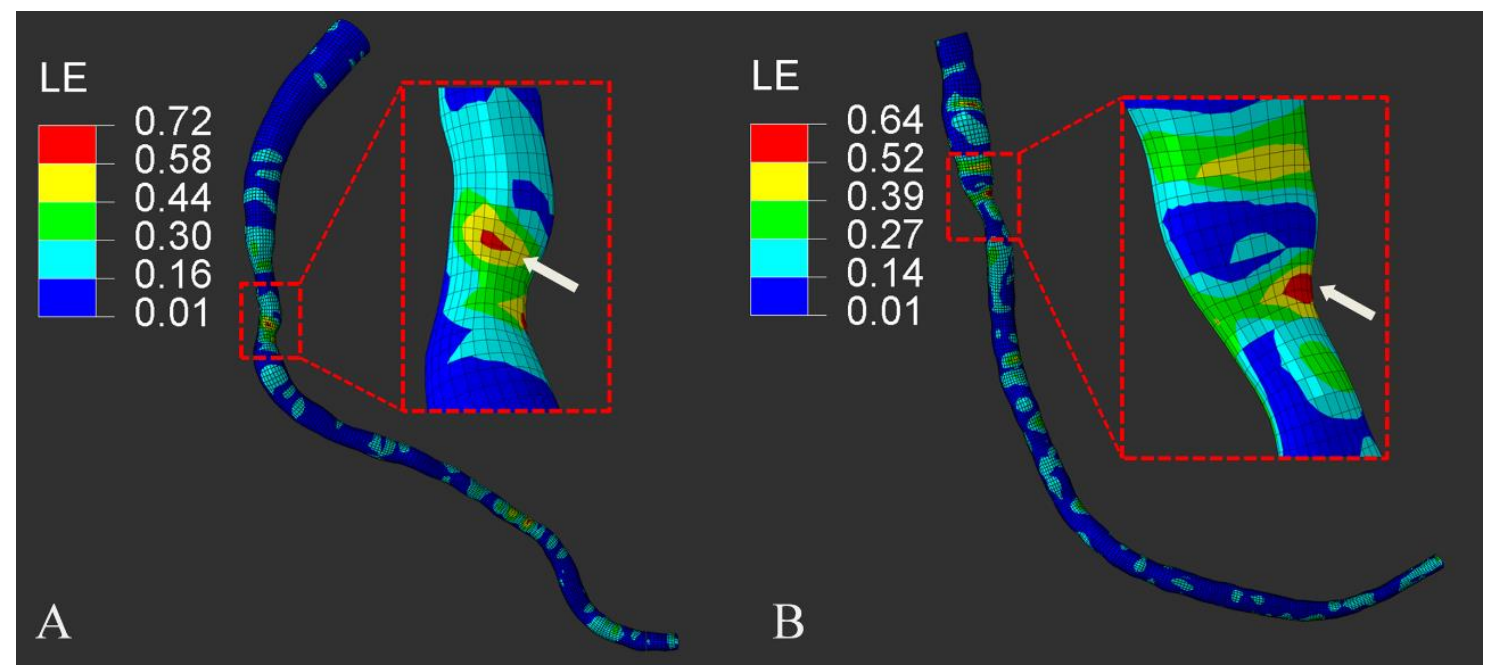

Strain distribution of both cases at corresponding phases of peak stress at baseline.

A) LAD; B) LCx.

White arrows indicate strain concentration. Enlarged graphs show spots of strain concentration consistent with sites of subsequent plaque rupture.

LE: maximum principal logarithmic strain

\section{Supplementary Appendix 2. Additional results}

When the thickness of the superficial wall was changed to five times thinner or thicker (i.e., $12 \mu \mathrm{m}$ and $300 \mu \mathrm{m}$ ), the pattern of dynamic SWS distribution remained unchanged and the peak SWS showed only slight changes to $114.0 \mathrm{kPa}$ and $124.7 \mathrm{kPa}$ in case 1 , and $95.23 \mathrm{kPa}$ and $102.0 \mathrm{kPa}$ in case 2, respectively. 


\section{Supplementary Appendix 3. Limitations}

Our approach is limited by the limited spatial resolution of coronary angiography. However, as modern X-ray angiograms with digital panels generally acquire images with higher resolution $(<0.1 \mathrm{~mm} /$ pixel $)$ but export images with lower resolution (typically around $0.2 \mathrm{~mm} /$ pixel) for post-processing, use of raw images might improve the accuracy of SWS computation. Although intravascular ultrasound (IVUS) and optical coherence tomography (OCT) have higher image resolutions, it is highly challenging to reconstruct true time-varying 4D coronary geometries based on these data. Furthermore, the application of the Mooney-Rivlin constitutive model was simple but is commonly used to describe hyperelastic material. Different constitutive model and corresponding material parameters can alter the result of stress distribution to some extent. Nevertheless, this has limited effects on the cyclic deformation and strain of the superficial arterial wall layer. Anisotropic constitutive models, such as Fung or Holzapfel models [9], may be used for the quantitative analysis of SWS at different directions. In addition, mechanical properties of fibrous caps may change over time due to temporary inflammation or therapy-induced reduction thereof (e.g., after aggressive lipid lowering). This may limit the value of SWS for predicting highrisk lesions. Finally, the accuracy of our proposed method requires a thorough validation before clinical applications can be considered. 